\title{
An antimicrobial stewardship elective for internal medicine residents
}

\author{
J. Patrik Hornak MD ${ }^{1}$ (1), Lindsay K. Sonstein $\mathrm{MD}^{1}$ and David Reynoso MD, $\mathrm{PhD}^{2}$ \\ ${ }^{1}$ Department of Internal Medicine, University of Texas Medical Branch, Galveston, Texas and ${ }^{2}$ Division of Infectious Diseases, University of Texas Medical Branch, \\ Galveston, Texas
}

To the Editor - Clinician education is a core element of antimicrobial stewardship programs (ASPs). ${ }^{1}$ Ideally, this education will occur early in a physician's career to maximally impact future behaviors. ${ }^{2}$ Many resident trainees are uncomfortable with antimicrobial stewardship concepts, as evidenced by a recent survey of fourth-year medical students, which revealed that $90 \%$ of them desired additional education regarding antibiotic appropriateness. ${ }^{3}$ Thus, with great interest we read the report by Childs-Kean et $\mathrm{al}^{4}$ reviewing the limited body of literature on medical and pharmacy trainee involvement with stewardship. In particular, just 2 reports describe resident involvement in antimicrobial stewardship. ${ }^{5,6} \mathrm{We}$ were unable to locate any publications outlining a dedicated elective course or clerkship in antibiotic stewardship for medical residents. Recently, we pilot tested such an elective at our institution. Motivated by the desire to meet an important, unfilled educational need while stimulating trainees' career interest in infectious diseases (ID), hospital epidemiology, and quality improvement (QI), and simultaneously aligning with institutional goals for optimizing antibiotic use as part of broader strategy for achieving high-value patient care, we created a comprehensive, 4-week elective in antimicrobial stewardship for internal medicine residents.

When designing our curriculum, we echoed sentiments by others that antibiotic stewardship should not rely solely on passive learning. ${ }^{1}$ Accordingly, our course emphasizes active learning along with a range of didactic components. With appropriate supervision by our permanent antibiotic stewards, including the clinical and pharmacist infectious diseases leaders, residents are incorporated into all major facets of the ASP's ongoing functions. These activities include daily participation with prospective audit-and-feedback and antibiotic time-outs, face-to-face interaction with treatment teams, attendance at multidisciplinary ASP meetings, and monitoring for early intervention opportunities in time-sensitive infectious conditions (eg, staphylococcal or extended-spectrum $\beta$-lactamase (ESBL)-producing gram-negative bacteremia). Upon completion of the elective, trainees are expected to have located an interesting topic for further discussion and to provide a 30-minute "resident talk" for their colleagues. Highly motivated trainees are welcomed to contribute to our ASP's longitudinal educational initiatives (eg, developing institutional guidelines for common infectious syndromes) and to participate in QI or other scholarly projects. Finally, we believe that this elective's emphasis on and immersion in prospective audit-andfeedback activities can uniquely augment residents' consultative skills, benefitting them well beyond the perimeter of a 1-month rotation.

Author for correspondence: Dr David Reynoso, Division of Infectious Diseases, University of Texas Medical Branch, 301 University Blvd, Rte 0435, Marvin Graves Building 4.210H, Galveston, TX 77555-0435. E-mail: dareynos@utmb.edu

Cite this article: Hornak JP, Sonstein LK, and Reynoso D. (2019). An antimicrobial stewardship elective for internal medicine residents. Infection Control \& Hospital Epidemiology, 40: 1212-1213, https://doi.org/10.1017/ice.2019.212
Certainly, we recognize that an effective antimicrobial steward needs a requisite level of knowledge to perform tasks such as recommending antimicrobial de-escalation, discontinuation, or dosing optimization. These tasks are often relegated exclusively to fellowship training. We also realize that not all residents have equivalent levels of confidence or ability. To meet the diverse needs of our learners, we created a complementary didactic curriculum for appropriate proficiency development. It includes assigned selections of high-quality learning modules from the Centers for Disease Control's Antibiotic Stewardship Training Series and videos from Wake Forest University's Antimicrobial Stewardship Curriculum, both of which are freely available online. ${ }^{7,8}$ These materials address the scientific and epidemiologic issues of antibiotic resistance, common scenarios of inappropriate antibiotic use, and tailored stewardship strategies for various clinical situations including ambulatory and inpatient settings. Additional knowledge is acquired through literature selections highlighting core stewardship concepts such as short course treatment for community-acquired pneumonia, avoidance of antibiotics in asymptomatic bacteriuria and acute bronchitis or rhinosinusitis, the effects of clindamycin restriction on Clostridium difficile infection rates, a review of the evidence for nephrotoxicity from concomitant use of vancomycin and piperacillin-tazobactam, the role of ID consultation in staphylococcal bacteremia, and penicillin allergy testing, among other topics. We also included readings highlighting situations for "antimicrobial optimization" such as escalation to a carbapenem for a bloodstream infection caused by an ESBL-producing isolate. Trainees are encouraged to rigorously employ clinical practice guidelines and high-quality evidence in guiding their antibiotic audits. Initially, we plan to restrict the elective to second- and third-year residents before considering extension to include first-year trainees. Lastly, when encountering confusing or complex clinical scenarios, residents are reminded to discuss various options with the ASP team before rendering interventions or to recommend formal ID consultations when appropriate.

We are excited about our new antimicrobial stewardship elective. As we pilot test this elective with interested residents, we look forward to assessing long-term impacts on knowledge retention, prescribing behavior, and career pursuits plus objective measurement of its contributions to the stewardship program, itself. The need to amplify antimicrobial stewardship education efforts for physician trainees and students cannot be overstated, as highlighted by Childs-Kean et al. ${ }^{4}$ Recent traction is evidenced by the development of a curricular framework in antimicrobial stewardship for ID fellows as developed by an Infectious Diseases Society of America taskforce. ${ }^{9}$ We agree with ChildsKean et al that the growing, broad need for antimicrobial stewardship may overwhelm the supply of formally trained personnel. Educational initiatives such as ours can help to bridge that gap by stimulating career interest and creating a sort of "stewardship 
extender." Although we have described a program for internal medicine residents, this curriculum can be easily adapted to other specialties. Lastly, we believe that stewardship initiatives in graduate medical education should complement innovative, like-minded programs at the medical school level. ${ }^{8,10}$ Educating the clinicians of tomorrow on the topic of antimicrobial stewardship is an area of limitless opportunity and impact. We encourage our colleagues to continue to explore and share new ways of accomplishing this vital task in the fight against antibiotic resistance.

Acknowledgments. We would like to thank our clinical ID pharmacist, Scott Ferren, PharmD, for his assistance with supporting this educational initiative.

Financial support. No financial support was provided relevant to this article.

Conflicts of interest. All authors report no conflicts of interest relevant to this article.

\section{References}

1. Barlam TF, Cosgrove SE, Abbo LM, et al. Implementing an antibiotic stewardship program: guidelines by the Infectious Diseases Society of America and the Society for Healthcare Epidemiology of America. Clin Infect Dis 2016;62(10):e51-e77.
2. Schwartz BS, Armstrong WS, Ohl CA, Luther VP. Create Allies, IDSA stewardship commitments should prioritize health professions learners. Clin Infect Dis 2015;61:1626-1627.

3. Abbo LM, Cosgrove SE, Pottinger PS, et al. Medical students' perceptions and knowledge about antimicrobial stewardship: how are we educating our future prescribers? Clin Infect Dis 2013;57:631-638.

4. Childs-Kean LM, Briggs HL, Cho JC. All aboard! Involvement of medical and pharmacy trainees in antimicrobial stewardship. Infect Control Hosp Epidemiol 2018. doi: 10.1017/ice.2018.332.

5. Lee TC, Frenette C, Jayaraman D, et al. Antibiotic self-stewardship: traineeled structured antibiotic time-outs to improve antimicrobial use. Ann Intern Med 2014;161 suppl 10:S53-S58.

6. Foral PA, Anthone JM, Destache CJ, et al. Education and communication in an interprofessional antimicrobial stewardship program. J Am Osteopath Assoc 2016;116:588-593.

7. CDC antibiotic stewardship training series. CDC Train website. https:// www.train.org/cdctrain/training_plan/3697. Published April 6, 2019. Accessed July 8, 2019.

8. Luther VP, Ohl CA, Hicks LA. Antimicrobial stewardship education for medical students. Clin Infect Dis 2013;57:1366.

9. Luther VP, Shnekendorf R, Abbo LM, et al. Antimicrobial stewardship training for infectious diseases fellows: program directors identify a curriculum need. Clin Infect Dis 2018;67:1285-1287.

10. Shekarchian S, Schwartz BS, Teherani A, et al. Is it time for a coordinated and longitudinal approach to antibiotic stewardship education? Clin Infect Dis 2016;63:848-849. 